\title{
Relação entre Obesidade e Diabete Mellitus Tipo II em Adultos
}

\section{Relationship between Obesity and Diabetes Mellitus Type II in Adults}

\author{
Fernanda de Almeida Escobar ${ }^{1}$
}

Artigo

Original

Original

Paper

\author{
Palavras-chaves: \\ Obesidade e \\ Sobrepeso \\ Diabetes Mellitus \\ tipo II
}

Gordura Corporal.

\section{Resumo}

Os maus hábitos alimentares e o estilo de vida estão associados a diversos prejuízos à saúde, entre eles, a obesidade. A obesidade tem sido apontada como um dos principais fatores de risco para o diabetes tipo 2. Estima-se que entre 80 e $90 \%$ dos indivíduos acometidos por essa doença são obesos. É observado um maior IMC e maior número de obesos dentre os indivíduos com algum grau de anormalidade da homeostase glicêmica. Com o aumento do IMC, o risco de se desenvolver diabetes fica mais pronunciado, contudo, o tecido adiposo atua aumentando a demanda por insulina em pacientes obesos, criando resistência a esta, o que ocasiona aumento na glicemia e consequente hiperinsulinemia. Pode-se conclui, com o presente estudo, que existe a correlação entre obesidade e diabetes tipo 2 , com isso, recomendase a perda de peso e a adoção de estilos de vida saudáveis.

\begin{abstract}
Poor diet and lifestyle are associated with several health hazards, including obesity. Obesity has been identified as one of the major risk factors for diabetes type 2. It is estimated that between 80 and $90 \%$ of individuals affected by this disease are obese. It is observed a higher BMI and higher obesity rate among individuals with some degree of abnormal glucose homeostasis. With increasing BMI, the risk of developing diabetes is more pronounced, however, adipose tissue acts to increase the demand for insulin in obese patients, creating resistance to it, which leads to increased blood glucose levels and consequent hyperinsulinemia. It can be concluded, with this study, that there is a correlation between obesity and diabetes type 2, with this, it is recommended that weight loss and adoption of healthy lifestyles.
\end{abstract}

Key words:

Obesity and

Overweight

Diabetes mellitus type II

Fat

\section{Introdução}

No Brasil, tem sido detectada a progressão da transição nutricional, caracterizada pela redução na prevalência dos déficits nutricionais e ocorrência mais expressiva de sobrepeso e obesidade, não só na população adulta, mas também em crianças e adolescentes (TRICHES, 2005). Segundo teorias ambientalistas, as causas estão fundamentalmente li- gadas às mudanças no estilo de vida e aos hábitos alimentares. Confirmando essas teorias, verifica-se que a obesidade é mais frequente em regiões mais desenvolvidas do País (Sul e Sudeste), pelas mudanças de hábitos associadas a esse processo (ESCRIVÃO et al. 2000). Os maus hábitos alimentares estão associados a diversos prejuízos à saúde, entre eles, a obesidade, cujos índices têm crescido nas últimas décadas, como resultado de aumento no con-

${ }^{1}$ Acadêmica do Curso de Nutrição - UniFOA 
sumo de alimentos com alta densidade calórica e redução na atividade física, ocorrendo assim, um problema de saúde pública. Há demonstrações de que, entre outros diversos fatores, o tempo que uma pessoa passa assistindo à TV pode estar associado à obesidade, visto que o mesmo exerce grande influência sobre os hábitos alimentares e promove o sedentarismo, pois cada hora diante da TV pode resultar em aumento de até $2 \%$ em sua prevalência da obesidade (ALMEIDA et al, 2002).

Sobrepeso e obesidade são importantes preocupações em saúde pública devido, principalmente, à sua associação com aumento de risco para hipertensão arterial sistêmica anormalidades lipídicas, diabetes mellitus e doença coronariana (MELLO et al, 2004). O elevado peso corporal, resultado do desequilíbrio entre oferta e demanda energética, e sua determinação tem-se revelado complexo e variável, em diversos aspectos, como fatores demográficos, socioeconômicos, genéticos, psicológicos, ambientais e individuais (TERRES et al. 2006).

A obesidade e o sobrepeso estão presentes na maioria dos pacientes diabéticos tipo 2 , sendo que sua prevalência varia dependendo de fatores genéticos e ambientais (educacionais e culturais) (CORRÊA et al. 2003). O diabetes mellitus é uma doença caracterizada por altas taxas de glicose e lipídios no sangue; essa glicose é transportada no sangue até as células pela insulina, onde será usada como fonte de energia. Quando se tem diabetes tipo II, o pâncreas não produz insulina suficiente, ou a insulina produzida não funciona adequadamente, com isso ocorre o aumento da quantidade de glicose no sangue (KRAUSE, 2002).

Devido ao aumento da incidência de diabetes mellitus tipo 2, principalmente em adultos obesos, foi observada a necessidade de realizar o presente estudo, com o objetivo de analisar a correlação entre a obesidade e diabete mellitus tipo 2 nessa faixa etária.

\section{Metodologia}

Este estudo trata-se de uma revisão bibliográfica baseada em artigos científicos, livros, sites atuais da Internet que abrangem o tema proposto, entre os anos 2000 a 2006.

\section{Referencial Teórico}

\subsection{Obesidade e Diabetes mellitus tipo II}

A crescente substituição dos alimentos in natura ricos em fibras, vitaminas e minerais, por produtos industrializados, associada a um estilo de vida sedentário, favorecido por mudanças na estrutura de trabalho e avanços tecnológicos, compõem um dos principais fatores etiológicos da obesidade (SARTORELLI, 2003).

A obesidade é uma doença metabólica crônica caracterizada pelo excesso de gordura corporal (GC). Existem vários métodos utilizados para a avaliação desse excesso, sendo mais usado o índice de massa corpórea (IMC). A obesidade é definida como IMC igual ou maior a $30 \mathrm{~kg} / \mathrm{m}^{2}$ e caracteriza-se pelo excesso de GC em relação à massa magra.

A obesidade tem sido apontada como um dos principais fatores de risco para o diabetes tipo 2. Estima-se que entre 80 e $90 \%$ dos indivíduos acometidos por esta doença são obesos e o risco está diretamente associado ao aumento do índice de massa corporal (SARTORELLI, 2003).

De acordo com Terres et al (2006), as consequências da obesidade têm sido relatadas em diversos trabalhos. O excesso de gordura em adultos está associado a maior ocorrência de Diabetes Mellitus, à hipertensão, ao aumento do triglicerídeo e do colesterol.

Segundo Martins \& Marinho (2003), a obesidade, principalmente a visceral, é o mais grave fator de risco cardiovascular e de distúrbio na homeostase glicoseinsulina. Resultando em várias alterações fisiopatológicas como a menor extração de insulina pelo fígado, com aumento da produção hepática de glicose e diminuição da captação de glicose pelo tecido muscular. Esses eventos podem resultar em diferentes graus de intolerância à glicose e, nos indivíduos com DM2, irão influenciar o controle glicêmico, refletido por maiores níveis de hemoglobina glicosilada (HbA1c). Esse fato deve ser analisado no contexto dos recentes estudos os quais demonstraram que nesses pacientes o controle glicêmico é fundamental para a redução do risco de evolução para complicações microvasculares (CORRÊE, et al. 2003). 
A frequência das complicações crônicas do DM do tipo 2 varia de acordo com as populações estudadas. Os pacientes com DM do tipo 2 têm uma propensão duas a quatro vezes maior de morrer por doença cardíaca em relação a não diabéticos, e quatro vezes mais chance de ter doença vascular periférica (DVP) e acidente vascular cerebral (AVC). Entre os fatores envolvidos na etiologia das complicações crônicas do DM do tipo 2, destacam-se a hiperglicemia, a hipertensão arterial sistêmica, a dislipidemia e o tabagismo (SCHEFFEL, et al. 2004).

É provável que esses aumentos na prevalência de diabetes decorram do já estabelecido aumento na prevalência de obesidade no Brasil. O elevado número de obesos na população, atualmente estudada em ambos os sexos, reforça essa hipótese. Além disso, foram observados maior IMC e maior número de obesos dentre os indivíduos com algum grau de anormalidade da homeostase glicêmica (diabetes ou glicemia de jejum alterada) (SCHAAN, 2004).

Francischi (2000) relata, em seu trabalho, que, para aumento de $10 \%$ no peso corporal, há aumento de $2 \mathrm{mg} / \mathrm{dl}$ na glicemia em jejum. Considerando a distribuição da gordura corporal, a circunferência da cintura maior do que $100 \mathrm{~cm}$ pode isoladamente elevar o risco do desenvolvimento de diabetes em 3,5 vezes, mesmo após um controle do IMC. No desenvolvimento de diabetes, o tecido adiposo atua aumentando a demanda por insulina e, em pacientes obesos, criando resistência a esta, o que ocasiona aumento na glicemia e consequente hiperinsulinemia. Contudo, a sensibilidade do tecido adiposo à insulina pode permanecer alta, o que sugere que a lipogênese possa estar favorecida. Em alguns casos, essa resistência pode ser atribuída à diminuição na concentração de receptores de insulina, ou em falha no mecanismo de trânsito celular.

O Diabetes Prevention Program demonstrou que, após três anos de acompanhamento, os indivíduos alocados no grupo de mudança do estilo de vida (dieta e exercício) tiveram uma diminuição do risco de evoluir para o diabetes de $58 \%$ que foi significativamente superior (58\% vs. $31 \%$ ) aos que utilizaram medicamentos (metformina). A presença de sobrepeso e obesidade exerce uma influência considerável na elevada morbidade e mortalidade da doença decorrente principalmente da associação com a doença cardiovascular, que é a principal causa de mortalidade em pacientes com DM2.

Gomes et al (2006) relataram que esse fato foi enfatizado em recente trabalho, demonstrando que $7,9 \%$ das internações em um Hospital Universitário foram devidas ao diabetes e suas complicações, sendo as principais causas as doenças cardiovasculares. Relatos do Sistema Único de Saúde (DATASUS) mostram que o diabetes é a quinta indicação de hospitalização no Brasil e está entre as dez maiores causas de mortalidade no país. O acima exposto deve ser analisado no contexto das estimativas recentes da OMS, que projetam um aumento significativo do número de indivíduos com diabetes até o ano de 2030. Nessa data, espera-se um universo de aproximadamente 366 milhões de indivíduos diabéticos, dos quais aproximadamente $90 \%$ apresentarão DM2 (GOMES et al. 2006).

Silveira observou, em seus resultados, o aumento da glicemia, paralelo à elevação do valor do IMC, fato este relacionado a um aumento da resistência à insulina. No estudo realizado por Campbell e Gerich, mostrou que indivíduos com IMC entre 19 à $25 \mathrm{Kg} / \mathrm{m}^{2}$ possuíam sensibilidade normal à insulina. Todavia, pessoas com IMC entre 25 à $26 \mathrm{Kg} / \mathrm{m}^{2}$, há um decréscimo da sensibilidade à insulina (constatando o aumento da resistência à mesma).

\section{Conclusão}

Pode-se concluir, com o presente estudo, que existe a correlação entre a obesidade e a diabetes mellitus tipo II, pois, à medida que o indivíduo aumenta sua massa gorda, seus níveis glicêmicos também se elevam, aumentando o risco de desenvolvimento do diabetes tipo 2. Recomenda-se a perda de peso na tentativa de prevenir esse quadro, bem como a adoção de estilos de vida saudáveis com alimentação balanceada e a prática de exercícios físicos. 


\section{Referencias Bibliográficas}

ALMEIDA, S. S. NASCIMENTO, P. C. B. D. QUAIOTI, T. C. B. Quantidade e qualidade de produtos alimentícios anunciados na televisão brasileira. Rev. Saúde Pública. São Paulo. vol.36 no.3. Jun 2002.

CORRÊA, F. H. S. et al. Influência da Gordura Corporal no Controle Clínico e Metabólico de Pacientes Com Diabetes Mellitus Tipo 2. Arq Bras Endocrinol Metab. Vol. 47. n1. Fev. 2003

ESCRIVÃO, M. A. M. S. et al. Obesidade exógena na infância e na adolescência. Jornal de Pediatria. Rio de Janeiro. vol 76. Suppl 3. p.305-10. 2000.

FRANCISCHI, R. P. P. Obesidade: atualização sobre sua etiologia, morbidade e tratamento. Rev. Nutr. Campinas. vol. 13. n1. p. 17-28. jan/abr. 2000.

GOMES, M. B. et al. Prevalência de Sobrepeso e Obesidade em Pacientes Com Diabetes Mellitus do Tipo 2 no Brasil: Estudo Multicêntrico Nacional. Arq Bras Endocrinol Metab. vol 50 n 1. Fevereiro. p.136-144. 2006.

MAHAN, Kathlee L. Escott-Stump S. Krause: Alimentos, Nutrição e Dietoterapia. 10 ed. São Paulo: Roca, 2002, p.719.

MARTINS,I.S. MARINHO,S.P. O potencial diagnóstico dos indicadores da obesidade centralizada. Rev Saúde Pública. São Paulo. Vol.37 n.6. p.760-767. 2003.

MELLO, et al.Atendimento ambulatorial individualizado versus programa de educação em grupo:qual oferece mais mudanças de hábitos alimentares e de atividade física em crianças obesas? Jornal de Pediatria. Rio de Janeiro. vol 80.n6.2004.

\section{SARTORELLI, D. S. FRANCO, L. J. Tendências} do diabetes mellitus no Brasil: o papel da transição nutricional. Cad. Saúde Pública. Rio de Janeiro. Vol. 19. Sup.1. p. 29-36. 2003.
SCHAAN,B.D. HARZHEIM,E. GUS,I Perfil de risco cardíaco no diabetes mellitus e na glicemia de jejum alterada. Rev. Saúde Pública. São Paulo. vol.38 n.4. p.529-536. 2004

SCHEFFEL, R. S. et al. Prevalência de Complicações Micro e Macrovasculares e de seus Fatores de Risco em Pacientes com Diabetes Melito do Tipo 2 em Atendimento Ambulatorial. Rev Assoc Med Bras. Porto Alegre. Vol.50, n 3. p. 263-267.2004.

SILVEIRA, L. A. G Correlação entre Obesidade e Diabetes Tipo 2. Pós-graduação Latu-Sensu em FisiologiadoExercícioeAvaliação-Morfofuncional Universidade Gama Filho. Juiz de Fora.

TERRES, N. G. et al. Prevalência e fatores associados ao sobrepeso e à obesidade em adolescentes. Rev Saúde Pública. Vol 40. N 4. p.627-633. 2006.

TRICHES, R.M.GIUGLIANI,E.R.J.Obesidade, práticas alimentares e conhecimentos de nutrição em escolares. Rev. Saúde Pública. São Paulo. vol. 39. n4 ago. p. 541-547. 2005.

Endereço para Correspondência:

Acadêmica de Nutrição Fernanda de Almeida Escobar (Ciências da Saúde - UniFOA) e-mail: nandaescobar@yahoo.com.br

Centro Universitário de Volta Redonda Campus Três Poços

Av. Paulo Erlei Alves Abrantes, $n^{\circ} 1325$, Três Poços - Volta Redonda / RJ CEP: $27240-560$ 